\title{
Evaluation of the reliability of the maximum entropy method for reconstructing 3D and 4D NOESY-type NMR spectra of proteins
}

Yoshiki Shigemitsu $^{a}$, Teppei Ikeya ${ }^{\mathrm{a}, \mathrm{b}}$, Akihiro Yamamoto ${ }^{\mathrm{a}}$, Yuusuke Tsuchie ${ }^{\mathrm{a}}$, Masaki Mishima $^{\mathrm{a}, \mathrm{b}}$, Brian O. Smith ${ }^{\mathrm{c}}$, Peter Güntert ${ }^{\mathrm{a}, \mathrm{d}, \mathrm{e}}$ \& Yutaka Ito I,b,* $^{\mathrm{a}}$

${ }^{a}$ Department of Chemistry, Graduate school of Science and Engineering, Tokyo Metropolitan University, 1-1 Minami-Osawa, Hachioji-shi, Tokyo 192-0373, Japan

${ }^{b}$ CREST/Japan Science and Technology Agency (JST), 4-1-8 Honcho, Kawaguchi, Saitama 332-0012, Japan

'Institute of Molecular, Cell and Systems Biology \& School of Life Sciences, College of Medical, Veterinary \& Life Sciences, Joseph Black Building, University of Glasgow, Glasgow G12 8QQ, UK.

${ }^{d}$ Institute of Biophysical Chemistry, Center for Biomolecular Magnetic Resonance, Goethe University Frankfurt am Main, Max-von-Laue-Str. 9, 60438 Frankfurt am Main, Germany

${ }^{e}$ Frankfurt Institute for Advanced Studies, Ruth-Moufang-Str. 1, 60438 Frankfurt am Main, Germany.

*Corresponding author:

Tel: +81-42-677-2545, Fax: +81-42-677-2525 


\section{E-mail address: ito-yutaka@tmu.ac.jp (Y. Ito)}

Keywords:

Protein NMR, 4D NMR, NOESY, spectrum reconstruction, maximum entropy, nonlinear sampling

Abbreviations:

CS, compressed sensing; FT, Fourier transform; IRLS, iteratively reweighted leastsquares; IST, iterative soft thresholding; MaxEnt, maximum entropy; MDD, multidimensional decomposition; QME, quantitative maximum entropy, RMSE, root mean square error; RMSD, root mean square deviation. 


\begin{abstract}
Despite their advantages in analysis, 4D NMR experiments are still infrequently used as a routine tool in protein NMR projects due to the long duration of the measurement and limited digital resolution. Recently, new acquisition techniques for speeding up multidimensional NMR experiments, such as nonlinear sampling, in combination with non-Fourier transform data processing methods have been proposed to be beneficial for 4D NMR experiments. Maximum entropy (MaxEnt) methods have been utilised for reconstructing nonlinearly sampled multi-dimensional NMR data. However, the artefacts arising from MaxEnt processing, particularly, in NOESY spectra have not yet been clearly assessed in comparison with other methods, such as quantitative maximum entropy, multidimensional decomposition, and compressed sensing.

We compared MaxEnt with other methods in reconstructing 3D NOESY data acquired with variously reduced sparse sampling schedules and found that MaxEnt is robust, quick and competitive with other methods. Next, nonlinear sampling and MaxEnt processing were applied to 4D NOESY experiments, and the effect of the artefacts of MaxEnt was evaluated by calculating 3D structures from the NOE-derived distance restraints. Our results demonstrated that sufficiently converged and accurate structures (RMSD of 0.91 $\AA$ to the mean and $1.36 \AA$ to the reference structures) were obtained even with NOESY spectra reconstructed from $1.6 \%$ randomly selected sampling points for indirect dimensions. This suggests that 3D MaxEnt processing in combination with nonlinear sampling schedules is still a useful and advantageous option for rapid acquisition of highresolution 4D NOESY spectra of proteins.
\end{abstract}




\section{Introduction}

For the structure determination of small to medium size proteins by solution NMR, 3D NOESY experiments are usually analysed for the collection of NOE-derived distance restraints, even though assignment ambiguity due to degeneracy of ${ }^{1} \mathrm{H}$ resonances remains for one of the ${ }^{1} \mathrm{H}$ dimensions. Further separation of the ambiguous ${ }^{1} \mathrm{H}$ dimension with the chemical shifts of directly bound ${ }^{13} \mathrm{C}$ or ${ }^{15} \mathrm{~N}$ nuclei in $4 \mathrm{D}$ spectra is a straightforward way to resolve the degeneracy. However, 4D NOESY experiments are less commonly used due to the long duration of the measurement and limited digital resolution in indirectly observed dimensions because generally insufficient data points are acquired in order to keep the measurement time manageable. It would therefore be advantageous to be able to measure 4D NOESY spectra with good digital resolution in affordable measurement time.

NMR spectroscopy is an inherently insensitive technique, thus new acquisition schemes for speeding up multidimensional NMR experiments are demanded for dramatic improvements in both sensitivity and resolution. Among the various approaches, nonlinear sampling for indirectly acquired dimensions (also called non-uniform sampling or sparse sampling) [1-3] has been shown to be a robust technique. The effect of reduced sampling schemes on structure determination has also been assessed [4].

Since discrete Fourier transform (FT) cannot be used for processing sparsely sampled data, maximum entropy (MaxEnt) [5,6] has been used as an alternative for more than 20 years. Recently, multi-dimensional decomposition (MDD) [7], non-uniform Fourier transform [8,9] and forward maximum-entropy reconstruction [10,11] have been proposed. More recently, $l_{p}$-norm $(0<p \leq 1)$ minimisation referred to as Compressed Sensing (CS) was introduced to the NMR field $[12,13]$. We have reported an extended version of MaxEnt based on the MemSys5 package [14], quantitative maximum entropy 
(QME) [15]. For the reconstruction of 4D NMR spectra, MDD [16], CLEAN [17,18], MaxEnt [19,20], and Iterative Soft Thresholding (IST) [21] have been applied.

One of the major criticisms to non-FT methods is their questionable reliability in reproducing cross peaks with proper signal intensity, especially in the case of signals with a wide dynamic range as in NOESY-type experiments. However, it has not yet been clearly compared the quality of "classical" MaxEnt processing in NOESY spectra with that of other methods.

In this report, we applied MaxEnt processing to $3 \mathrm{D}{ }^{15} \mathrm{~N}$-separated and ${ }^{13} \mathrm{C}$-separated NOESY of a small protein, the Thermus thermophilus HB8 TTHA1718 gene product, and compared its reliability in reproducing accurate signal intensity from nonlinearly sampled data with the alternative approaches MDD, CS, and QME. In addition, we employed a nonlinear sampling scheme in $4 \mathrm{D}{ }^{13} \mathrm{C} /{ }^{15} \mathrm{~N}$-separated and ${ }^{13} \mathrm{C} /{ }^{13} \mathrm{C}$-separated NOESY of TTHA1718 and assessed the quality of MaxEnt processing on these 4D NOESY data by calculating 3D structures from the NOE-derived distance restraints obtained from the reconstructed spectra. 


\section{Materials and Methods}

Sample preparation and NMR spectroscopy

The expression and purification of ${ }^{13} \mathrm{C} /{ }^{15} \mathrm{~N}$-labelled TTHA1718 were performed as described previously [22]. The final ${ }^{13} \mathrm{C} /{ }^{15} \mathrm{~N}$-TTHA1718 fractions were concentrated to approximately $1.0 \mathrm{mM}$ and dissolved in $\mathrm{M} 9$ medium containing $10 \% \mathrm{D}_{2} \mathrm{O}$ for NMR lock.

NMR experiments were performed at $37^{\circ} \mathrm{C}$ probe temperature in a triple-resonance cryoprobe fitted with a z-axis pulsed field gradient coil, using a Bruker Avance $600 \mathrm{MHz}$ spectrometer. The 3D ${ }^{15} \mathrm{~N}$-separated and ${ }^{13} \mathrm{C}$-separated NOESY experiments were measured with 80 ms NOE mixing time and a total of $512\left(t_{3},{ }^{1} \mathrm{H}\right.$ acquisition $) \times 128\left(t_{1}\right.$, $\left.{ }^{1} \mathrm{H}\right) \times 32\left(t_{2},{ }^{15} \mathrm{~N}\right.$ or $\left.{ }^{13} \mathrm{C}\right)$ complex points. The $4 \mathrm{D}{ }^{13} \mathrm{C} /{ }^{15} \mathrm{~N}$-separated and ${ }^{13} \mathrm{C} /{ }^{13} \mathrm{C}$-separated NOESY experiments were measured with 8 transients, a 200 ms NOE mixing time and a total of $512\left(t_{4},{ }^{1} \mathrm{H}^{\mathrm{N}}\right.$ acquisition $) \times 32\left(t_{1},{ }^{1} \mathrm{H}\right) \times 24\left(t_{2},{ }^{13} \mathrm{C}\right) \times 8\left(t_{3},{ }^{15} \mathrm{~N}\right)$ and $512\left(t_{4},{ }^{1} \mathrm{H}\right.$ acquisition $) \times 24\left(t_{1},{ }^{1} \mathrm{H}\right) \times 20\left(t_{2}{ }^{13} \mathrm{C}\right) \times 18\left(t_{3}{ }^{13} \mathrm{C}\right)$ complex points, respectively. The total measurement times for the $4 \mathrm{D}{ }^{13} \mathrm{C} /{ }^{15} \mathrm{~N}$-separated and $4 \mathrm{D}{ }^{13} \mathrm{C} /{ }^{13} \mathrm{C}$-separated NOESY experiments were 5.7 and 6.4 days, respectively. These 3D and 4D NOESY data are henceforth referred to as "reference” data.

In order to achieve nonlinear sampling, the pulse sequences were modified according to the procedure reported by Rovnyak et al.[3]. The pulse programs with conventional and nonlinear sampling and the VC list generator program are available from the corresponding author.

\section{Preparation of various data sets with conventional and nonlinear sampling}

For the evaluation of the artefacts arising from the employment of nonlinear sampling and MaxEnt processing, data sets with various randomly sampled points in the indirect 
dimensions were prepared from the reference 3D and 4D NOESY data. For the nonlinearly sampled data, sampling schemes were generated using an in-house program. Six steps for random reduction of sampling points, 1/2, 1/4, 1/8, 1/16, 1/32 and 1/64, were generated for 3D NOESY experiments, while seven steps of sampling points, 1/2, 1/4, 1/8, 1/16, 1/32, 1/64 and 1/128 were generated for 4D NOESY experiments. In order to assess the deviation due to the selected sampling points, three different sampling schedules were generated from different random seeds for each random reduction step. Next, new data sets were concatenated by rearranging the raw data based on the schedules. These 3D and 4D NOESY data are henceforth referred to as "nonlinearly sampled” data.

For comparison, conventionally (linearly) sampled $4 \mathrm{D}{ }^{13} \mathrm{C} /{ }^{15} \mathrm{~N}$-separated and 4D ${ }^{13} \mathrm{C} /{ }^{13} \mathrm{C}$-separated NOESY data sets with reduced numbers of data points were also prepared corresponding to approximately 1/2,1/4,1/8,1/16, 1/32 and 1/128 of the reference data sets. These 4D NOESY data are henceforth referred to as "linearly sampled" data. The parameters, e.g. total number of data points for all indirect dimensions, are described in Supplementary Tab. S1.

\section{Data processing and spectral analysis}

The reference, nonlinearly sampled and linearly sampled 3D and 4D NOESY data were processed with 2D and 3D MaxEnt, respectively, on LINUX-PCs using the AZARA 2.7/2.8 software suite (W. Boucher, http://www.bio.cam.ac.uk/azara/). Consequently, 3D NOESY spectra were produced with $512\left(F_{3},{ }^{1} \mathrm{H}\right) \times 512\left(F_{1},{ }^{1} \mathrm{H}\right) \times 128\left(F_{2},{ }^{13} \mathrm{C}\right.$ or $\left.{ }^{15} \mathrm{~N}\right)$ data points, and 4D ${ }^{13} \mathrm{C} /{ }^{15} \mathrm{~N}$-separated NOESY and $4 \mathrm{D}{ }^{13} \mathrm{C} /{ }^{13} \mathrm{C}$-separated NOESY spectra were produced with $512\left(F_{4},{ }^{1} \mathrm{H}^{\mathrm{N}}\right) \times 128\left(F_{1},{ }^{1} \mathrm{H}\right) \times 128\left(F_{2},{ }^{13} \mathrm{C}\right) \times 64\left(F_{3},{ }^{15} \mathrm{~N}\right)$, and 480 
$\left(F_{4},{ }^{1} \mathrm{H}\right) \times 128\left(F_{1},{ }^{1} \mathrm{H}\right) \times 128\left(F_{2},{ }^{13} \mathrm{C}\right) \times 128\left(F_{3},{ }^{13} \mathrm{C}\right)$ data points, respectively. The duration of 3D MaxEnt processing depends upon the number of iterations, the sizes of both input and output data, etc. Typically the processing took 10-20 minutes for 3D ${ }^{13} \mathrm{C} /{ }^{15} \mathrm{~N}$-separated NOESY and $6-16$ hours for $4 \mathrm{D}{ }^{13} \mathrm{C} /{ }^{13} \mathrm{C}$-separated NOESY data using a LINUX-PC with a $3.4 \mathrm{GHz}$ Intel Core i7-4770 CPU. In addition, the "reference" 3D and 4D spectra were also processed with conventional FT on Azara for all dimensions. For 4D Fourier transform, linear prediction was utilised for the indirect dimensions.

In order to assess the quality of MaxEnt-processed 3D NOESY data, MDD, CS [with IST and iteratively reweighted least-squares (IRLS) algorithms], and QME processing were employed for comparison.

The MDD and CS processings were performed by the MDDNMR software [23] on the nmrPipe [24] platform. After processing the directly acquired dimension ( $\left.t_{3}\right)$ by FT using nmrPipe, MDD and CS calculations were performed by employing the standard parameters used in the example scripts of the software, and 3D interferograms were reconstructed with $256\left(t_{1}\right) \times 64\left(t_{2}\right)$ complex points for the indirect dimensions. The indirect dimensions were then apodised, zero-filled (x2) and processed with FT.

The QME processing was performed by a C-language program after processing the directly acquired dimension ( $\left.t_{3}\right)$ by FT using Azara.

All spectra were visualised and analysed on LINUX-PCs with the combination of customised macro programs on the OpenGL-version of ANSIG 3.3 software $[25,26]$ and the CcpNmr Analysis 2.2.2 software [27]. Peak positions were identified using the automated peak picking algorithm of Azara.

Structure calculation 
The structure calculations were performed with the program CYANA [28] version 3.0 using automated NOE assignment [29] and torsion angle dynamics for the structure calculation [30]. Assigned peak lists were converted from ANSIG into XEASY format as input data for the structure calculation. Backbone dihedral angle restraints obtained from chemical shifts with the program TALOS [31] were added to the input for CYANA. Structure calculations were performed simultaneously on 20 processors of a Linux cluster system with $3.07 \mathrm{GHz}$ Intel Core i7-950 processors. 


\section{Results}

Comparison of various methods for the reconstruction of 3D NOESY spectra

The comparison of a representative $F_{1}\left({ }^{1} \mathrm{H}\right)-F_{2}\left({ }^{15} \mathrm{~N}\right.$ or $\left.{ }^{13} \mathrm{C}\right)$ slice between the 3D NOESY spectra reconstructed by various methods is shown in Supplementary Figs. S1 (3D ${ }^{15} \mathrm{~N}$-separated NOESY) and S2 (3D ${ }^{13} \mathrm{C}$-separated NOESY). The root mean square error (RMSE) of the peak intensities against the spectra processed from the reference data by FT was employed for the validation of the spectra with the number of sampling points systematically reduced from $1 / 2$ to $1 / 16$ (Fig. 1). Since the number of picked peaks decreased with the reduction of sampling points, the RMSEs converged for all the methods to nearly identical values, -0.3 and -0.2 in ${ }^{13} \mathrm{C}$ - and ${ }^{15} \mathrm{~N}$-separated NOESY, respectively. These results show that the spectra processed by MaxEnt and QME had slightly better quality than those processed by MDD or IST, over almost the entire range of sampling data points. The IRLS-processed ${ }^{15} \mathrm{~N}$-separated NOESY spectra were better than those of MaxEnt and QME in the cases of all and 1/2 data points. QME somewhat outperformed MaxEnt, which is considered to be the effect of automatic estimation of the Lagrange multiplier, $\lambda$, in QME. On the other hand, in all cases the standard deviations were rather large and with overlap of the distributions of the data from the different methods, which required to evaluate the statistical significance of the spectrum qualities among them. Statistical hypothesis testing was thus performed by three standard approaches, the Student and Welch $t$-tests, and the Wilcoxon rank-sum test, which were selected according to normality and equality of variance. The $p$-values of the RMSEs with MaxEnt compared to MDD, IST and IRLS for the ${ }^{13} \mathrm{C} /{ }^{15} \mathrm{~N}$-selected NOESY spectra with 1/4 data points were sufficiently small for rejecting the null hypothesis that the two RMSEs are equal, except for that of the ${ }^{13} \mathrm{C}$-separated NOESY spectra between MaxEnt 
and IRLS, 0.244. These results show that, at least in our evaluation criteria, the quality of the spectra by MaxEnt are equivalent or even better than those from other processing methods. Hence MaxEnt can be one of the options for the reconstruction of 3D NOESY spectra even at present.

\section{D MaxEnt processing of the reference $4 D$ NOESY data}

3D MaxEnt processing of the two reference 4D NOESY spectra of TTHA1718 was performed, and nicely separated 4D spectra were obtained and analysed. On the basis of the collected NOE-derived distance restraints and with/without TALOS-derived backbone $\phi / \psi$ dihedral angle restraints the 3D structures of TTHA1718 were calculated three times repeatedly with different seed values (Supplementary Tab. S2). The average of these three runs showed well-converged ensemble of structures with a backbone root mean square deviation (RMSD) of $0.32 \pm 0.01 \AA$ (with dihedral angle restraints) to the mean coordinates, and is similar to the structure that was determined previously [22] with a backbone RMSD of $0.57 \pm 0.02 \AA$ (with dihedral angle restraints), indicating that the NOE cross peaks in the reference 4D NOESY spectra are sufficient for the 3D structure calculations with good accuracy and precession.

3D MaxEnt processing of the nonlinearly sampled and linearly sampled 4D NOESY data

Next nonlinearly sampled and linearly sampled 4D NOESY data with various numbers of sampling points were processed and analysed with the essentially identical protocols. Fig. 2 and Supplementary Fig. S3 show the comparison of a representative $F_{1}\left({ }^{1} \mathrm{H}\right)-F_{2}\left({ }^{13} \mathrm{C}\right)$ slice between the spectra. Obviously, in the linearly sampled spectra, line broadening of cross peaks becomes significant with decreasing the number of sampled data points. In 
contrast, in the nonlinearly sampled spectra, the line shaped remained similar even with much reduced numbers of sampled data points.

Intensities of NOE cross peaks in the MaxEnt-processed 4D NOESY spectra

Statistics of NOE-derived distance restraints collected for each representative data set are summarised in Supplementary Table S3. There was a tendency that the more data points were omitted, the fewer number of NOE cross peaks were picked. Nevertheless, when comparing the nonlinearly sampled spectra versus linearly sampled counterpart, more NOE cross peaks were observed in the nonlinearly sampled spectra, and this difference became significant when more data points were omitted. In order to examine this tendency, intensities of NOE cross peaks from various MaxEnt-processed 4D spectra were plotted against the intensity of the corresponding cross peaks in the reference spectra (Supplementary Figs. S4-S7). Since the absolute intensity of cross peaks decreased as the number of sampled data points was reduced, peak intensities were normalised relative to the reference cross peaks for each spectrum by an overall normalization factor given by the ratio of the average logarithms of peak intensities in both spectra. Generally speaking, the intensities of NOE cross peaks were fairly correctly reproduced from the MaxEntprocessed data sets. However, for weaker NOE cross peaks, the more data points were omitted, the more underestimated were the intensities. This presumably caused the drop of the numbers of picked NOE cross peaks in the spectra.

Structure calculations based on the NOE-derived distance restraints obtained in the MaxEnt-processed 4D NOESY spectra 
The effect of the artefacts arising by employing nonlinear sampling and 3D MaxEnt processing to 4D NOESY spectra was evaluated by calculating structures based on the NOE-derived distance restraints obtained in the MaxEnt-processed 4D NOESY spectra with various reduced numbers of sampling points. The calculations were performed with essentially the identical procedure as for the reference data set. Fig. 3 and Supplementary Fig. S8 show the superimposed 20 final conformers calculated from the NOE-derived distance restraints obtained in the MaxEnt-processed 4D NOESY spectra from nonlinearly sampled and linearly sampled data, respectively. The effect of adding backbone dihedral angle restraints from TALOS analysis was also compared in the figures. Structural statistics are summarised in Supplementary Tables S4-S7. Correlated with the decrease of the numbers of NOE cross peaks used, there was a tendency that the more data points were omitted, the less the ensembles of final structures converged. Backbone RMSD to the averaged coordinates of the reference structures increased for the structures calculated from the spectra reconstructed from much reduced numbers of sampling points. As was mentioned above, the intensities of relatively weaker NOE cross peaks were underestimated in the spectra reconstructed from the data with much reduced data points. In addition, there is a tendency that long-range NOEs, which are essential for the definition of the proper protein fold, are generally weaker than intra-residual and sequential NOEs. Therefore, it can be concluded that the reduction of sampling points decreased the relative percentage of long-range structural restraints, thus ultimately increasing the backbone RMSD to the reference structures. Nevertheless, we emphasise that, with the same number of data points, the convergence of the final structures from nonlinearly sampled data were much better than from linearly sampled data. Even with 1/64 data points, the MaxEnt-processed spectra could provide structures with fairly good 
agreement (backbone RMSD of $1.36 \AA$ to the reference coordinates) with the reference structure and with reasonable precision (backbone RMSD of $0.91 \AA$ to the average coordinates). 


\section{Discussion}

The comparison of various processing methods showed that the classical MaxEnt is still a robust, quick and competitive way to reconstruct 3D NOESY spectra. It is also remarkable that 3D MaxEnt successfully reconstructed 4D NOESY, particularly the 4D ${ }^{13} \mathrm{C} /{ }^{13} \mathrm{C}$-NOESY spectra. 4D ${ }^{13} \mathrm{C} /{ }^{13} \mathrm{C}$-NOESY spectra have large diagonal (selfcorrelated) cross peaks, and their large dynamic range in peak intensity have been thought to be problematic for MaxEnt processing.

The main artefacts arising from nonlinear sampling and non-FT processing were deviations of peak intensities, the loss of original cross peaks, and the emergence of false signals. The second problem is, to a certain extent, inevitable when utilising nonlinear sampling, since the reduction of total acquisition time causes a decrease of the signal-tonoise ratio of the data. The third problem is not likely to have happened since the MaxEnt iteration process with Azara starts from a "flat" spectrum. And even if it happened the false peak problem may not be significant because the automated NOE assignment algorithm implemented in CYANA excludes “orphan” artefact cross peaks. The first problem, the deviation of intensities for NOE cross peaks from their "real" values, is usually not harmful for structure calculations, since the NOE intensities are generally interpreted as distance restraints with conservatively set upper distance bounds. Because of the inverse sixth power intensity-to-distance relationship the relative error of the upper distance bounds is only one sixth of the relative error of the cross peak intensities. Indeed, structure calculations from distance restraints for which the $\pm 100 \%$ errors were introduced to the intensity of NOE cross peaks showed no significant changes in the structures (Supplementary Fig. S9 and Supplementary Table S8). From this point of view 3D MaxEnt is still a valuable option for the processing of nonlinearly sampled 4D 
NOESY data. However, the mis-calibration of intensities started having a significant effect to decrease the numbers of picked NOE cross peaks in the case of much reduced data points, e.g. in the case of 1/128 random sampling points, thereby affecting the accuracy of the calculated structures. Nevertheless, we emphasise that 4D NOESY with nonlinear sampling is very advantageous to rapid determination of accurate global folds, particularly for cases suffering from short sample life times or low sensitivity.

\section{Acknowledgments}

The authors thank Prof. Seiki Kuramitsu for providing the plasmid encoding TTHA1718, and Drs Daniel Nietlispach and Markus Wälchli for setting up 4D NOESY experiments with nonlinear sampling schemes. This work was supported in part by the Funding Program for Next Generation World-Leading Researchers (NEXT Program) from the Japan Society for the Promotion of Science (JSPS) and CREST from the Japan Science and Technology Agency (JST). 
Fig. 1. The quality of the reconstructions of ${ }^{15} \mathrm{~N}-$ (a) and ${ }^{13} \mathrm{C}-$ (b) separated NOESY spectra obtained by five different approaches: MaxEnt (red), QME (green), MDD (blue), IRLS (purple), and IST (light blue). The RMSE, shown on logarithmic scale, are the rootmean-square relative errors of the intensities of all picked peaks with respect to those of the FT-processed reference data. The lines, and up and down arrow marks show the average and range of standard deviations of the RMSE, respectively.

Fig. 2. Schematic illustration of the conventional (a) and the nonlinear sampling schemes with 1/2 (b), 1/4 (c), 1/8 (d), 1/16 (e), 1/32 (f), 1/64 (g), and 1/128 (h) randomly selected sampling points for $t_{1}, t_{2}$ and $t_{3}$ indirect dimensions of the $4 \mathrm{D}{ }^{13} \mathrm{C} /{ }^{15} \mathrm{~N}$-selected NOESY spectra. A representative $F_{1}\left({ }^{1} \mathrm{H}\right)-F_{2}\left({ }^{13} \mathrm{C}\right)$ cross section at $F_{4}\left({ }^{1} \mathrm{H}^{\mathrm{N}}\right)=7.68 \mathrm{ppm}$ and $F_{3}\left({ }^{15} \mathrm{~N}\right)=121.2 \mathrm{ppm}$ was extracted and shown for the MaxEnt processed $4 \mathrm{D}{ }^{13} \mathrm{C} /{ }^{15} \mathrm{~N}$ selected NOESY spectra reconstructed from the conventionally acquired reference data (i) and the data with 1/2 (j), 1/4 (k), 1/8 (l), 1/16 (m), 1/32 (n), 1/64 (o), and 1/128 (p) randomly selected sampling points. Similarly, a representative $F_{1}\left({ }^{1} \mathrm{H}\right)-F_{2}\left({ }^{13} \mathrm{C}\right)$ cross section at $F_{4}\left({ }^{1} \mathrm{H}\right)=6.74 \mathrm{ppm}$ and $F_{3}\left({ }^{13} \mathrm{C}\right)=117.85 \mathrm{ppm}$ (aliased) was extracted and shown for the MaxEnt processed 4D ${ }^{13} \mathrm{C} /{ }^{13} \mathrm{C}$-selected NOESY spectra reconstructed from the conventionally acquired reference data (q) and the data with 1/2 (r), 1/4 (s), 1/8 (t), 1/16 (u), 1/32 (v), 1/64 (w), and 1/128 (x) randomly selected sampling points.

Fig. 3. Superpositions of the 20 final structures of TTHA1718 calculated from distance restraints obtained from $4 \mathrm{D}{ }^{13} \mathrm{C} /{ }^{15} \mathrm{~N}$-separated and ${ }^{13} \mathrm{C} /{ }^{13} \mathrm{C}$-separated NOESY data with conventional sampling (a, i), and with the nonlinear samplings of $1 / 2(\mathbf{b}, \mathbf{j}), 1 / 4$ (c, $\mathbf{k})$ 1/8 (d, l), 1/16 (e, m), 1/32 (f, n), 1/64 (g, o), and 1/128 (h, p) randomly selected complex 
points for the indirect dimensions. The ensemble of structures shown in panels a-h (colour coded in blue) were calculated from the NOE-derived distance restraints and TALOS-derived dihedral angle restraints, while the backbone dihedral angle restraints were excluded for the calculations of the structures shown in panels i-p (colour coded in grey). The previously reported 20 final structures of TTHA1718 (PDB ID: 2ROE) calculated from the NOE-derived distance restraints from the $3 \mathrm{D}{ }^{15} \mathrm{~N}$-separated and ${ }^{13} \mathrm{C}$ separated NOESY with the conventional sampling are shown in red for comparison. 


\section{References}

[1] J.C.J. Barna, E.D. Laue, M.R. Mayger, et al., Exponentional sampling, an alternative method for sampling in two-dimensional NMR experiments, J. Magn. Reson., 73 (1987) 69-77.

[2] P. Schmieder, A.S. Stern, G. Wagner, J.A. Hoch, Improved resolution in tripleresonance spectra by nonlinear sampling in the constant-time domain, J. Biomol. NMR, 4 (1994) 483-490.

[3] D. Rovnyak, D.P. Frueh, M. Sastry, et al., Accelerated acquisition of high resolution triple-resonance spectra using non-uniform sampling and maximum entropy reconstruction, J. Magn. Reson., 170 (2004) 15-21.

[4] S. Tikole, V. Jaravine, V.Y. Orekhov, P. Güntert, Effects of NMR spectral resolution on protein structure calculation, PLoS One, 8 (2013) e68567.

[5] E.D. Laue, M.R. Mayger, J. Skiiling, J. Staunton, Reconstrution of phase sensitive 2D NMR spectra by maximum entropy., J. Magn. Reson., 68 (1986) 14-29.

[6] J.A. Hoch, A.S. Stern, NMR data processing, (1996).

[7] V.Y. Orekhov, I. Ibraghimov, M. Billeter, Optimizing resolution in multidimensional NMR by three-way decomposition, J. Biomol. NMR, 27 (2003) 165-173.

[8] K. Kazimierczuk, A. Zawadzka, W. Kozminski, I. Zhukov, Random sampling of evolution time space and Fourier transform processing, J. Biomol. NMR, 36 (2006) 157168.

[9] J. Stanek, W. Kozminski, Iterative algorithm of discrete Fourier transform for processing randomly sampled NMR data sets, J. Biomol. NMR, 47 (2010) 65-77. 
[10] S.G. Hyberts, D.P. Frueh, H. Arthanari, G. Wagner, FM reconstruction of nonuniformly sampled protein NMR data at higher dimensions and optimization by distillation, J. Biomol. NMR, 45 (2009) 283-294.

[11] S.G. Hyberts, G.J. Heffron, N.G. Tarragona, et al., Ultrahigh-resolution ${ }^{1} \mathrm{H}-{ }^{13} \mathrm{C}$ HSQC spectra of metabolite mixtures using nonlinear sampling and forward maximum entropy reconstruction, J. Am. Chem. Soc., 129 (2007) 5108-5116.

[12] K. Kazimierczuk, V.Y. Orekhov, Accelerated NMR spectroscopy by using compressed sensing, Angew. Chem. Int. Ed. Engl., 50 (2011) 5556-5559.

[13] D.J. Holland, M.J. Bostock, L.F. Gladden, D. Nietlispach, Fast multidimensional NMR spectroscopy using compressed sensing, Angew. Chem. Int. Ed. Engl., 50 (2011) 6548-6551.

[14] J. Skilling, Quantified Maximum-Entropy, American Laboratory, 24 (1992) J32M32.

[15] J. Hamatsu, D. O'Donovan, T. Tanaka, et al., High-resolution heteronuclear multidimensional NMR of proteins in living insect cells using a baculovirus protein expression system, J. Am. Chem. Soc., 135 (2013) 1688-1691.

[16] V. Tugarinov, W.Y. Choy, V.Y. Orekhov, L.E. Kay, Solution NMR-derived global fold of a monomeric 82-kDa enzyme, Proc. Natl. Acad. Sci. U S A, 102 (2005) 622-627. [17] B.E. Coggins, P. Zhou, High resolution 4-D spectroscopy with sparse concentric shell sampling and FFT-CLEAN, J. Biomol. NMR, 42 (2008) 225-239.

[18] B.E. Coggins, J.W. Werner-Allen, A. Yan, P. Zhou, Rapid protein global fold determination using ultrasparse sampling, high-dynamic range artifact suppression, and time-shared NOESY, J. Am. Chem. Soc., 134 (2012) 18619-18630. 
[19] M. Mobli, A.S. Stern, W. Bermel, et al., A non-uniformly sampled 4D HCC(CO)NHTOCSY experiment processed using maximum entropy for rapid protein sidechain assignment, J. Magn. Reson., 204 (2010) 160-164.

[20] A. Gautier, H.R. Mott, M.J. Bostock, et al., Structure determination of the sevenhelix transmembrane receptor sensory rhodopsin II by solution NMR spectroscopy, Nat. Struct. Mol. Biol., 17 (2010) 768-774.

[21] S.G. Hyberts, A.G. Milbradt, A.B. Wagner, et al., Application of iterative soft thresholding for fast reconstruction of NMR data non-uniformly sampled with multidimensional Poisson Gap scheduling, J. Biomol. NMR, 52 (2012) 315-327.

[22] D. Sakakibara, A. Sasaki, T. Ikeya, et al., Protein structure determination in living cells by in-cell NMR spectroscopy, Nature, 458 (2009) 102-105.

[23] V.Y. Orekhov, V.A. Jaravine, Analysis of non-uniformly sampled spectra with multi-dimensional decomposition, Prog. Nucl. Magn. Reson. Spectrosc., 59 (2011) 271292.

[24] F. Delaglio, S. Grzesiek, G.W. Vuister, et al., NMRPipe: a multidimensional spectral processing system based on UNIX pipes, J. Biomol. NMR, 6 (1995) 277-293.

[25] P.J. Kraulis, G.M. Clore, M. Nilges, et al., Determination of the three-dimensional solution structure of the C-terminal domain of cellobiohydrolase I from Trichoderma reesei. A study using nuclear magnetic resonance and hybrid distance geometrydynamical simulated annealing, Biochemistry, 28 (1989) 7241-7257.

[26] P.J. Kraulis, P.J. Domaille, S.L. Campbell-Burk, et al., Solution structure and dynamics of ras p21.GDP determined by heteronuclear three- and four-dimensional NMR spectroscopy, Biochemistry, 33 (1994) 3515-3531. 
[27] W.F. Vranken, W. Boucher, T.J. Stevens, et al., The CCPN data model for NMR spectroscopy: development of a software pipeline, Proteins, 59 (2005) 687-696.

[28] P. Güntert, Automated structure determination from NMR spectra, Eur. Biophys. J., 38 (2009) 129-143.

[29] T. Herrmann, P. Güntert, K. Wüthrich, Protein NMR structure determination with automated NOE assignment using the new software CANDID and the torsion angle dynamics algorithm DYANA, J. Mol. Biol., 319 (2002) 209-227.

[30] P. Güntert, C. Mumenthaler, K. Wüthrich, Torsion angle dynamics for NMR structure calculation with the new program DYANA, J. Mol. Biol., 273 (1997) 283-298. [31] G. Cornilescu, F. Delaglio, A. Bax, Protein backbone angle restraints from searching a database for chemical shift and sequence homology, J. Biomol. NMR, 13 (1999) 289_ 302. 
a

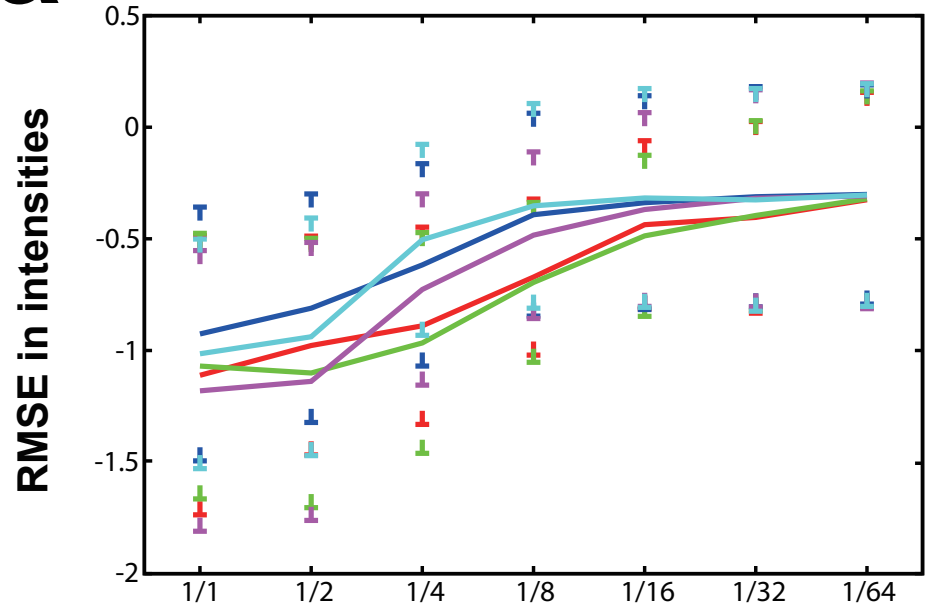

ratio of number of sampling points

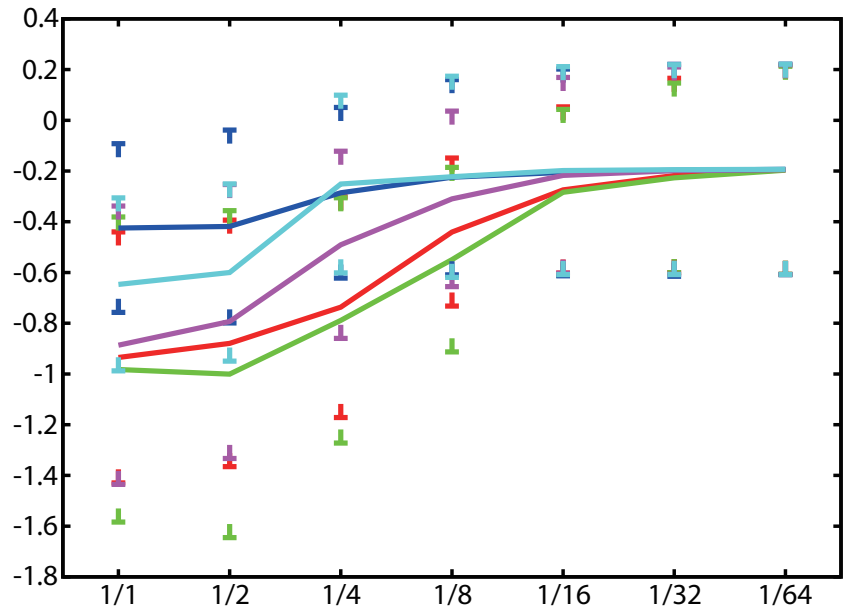

ratio of number of sampling points 
Figure 2

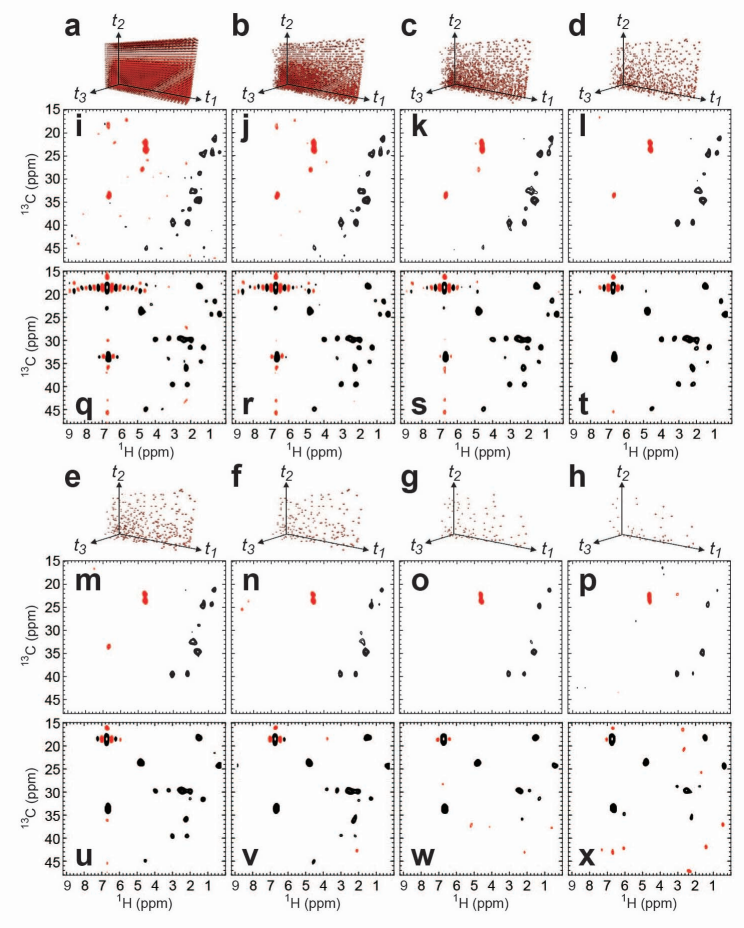


Figure 3
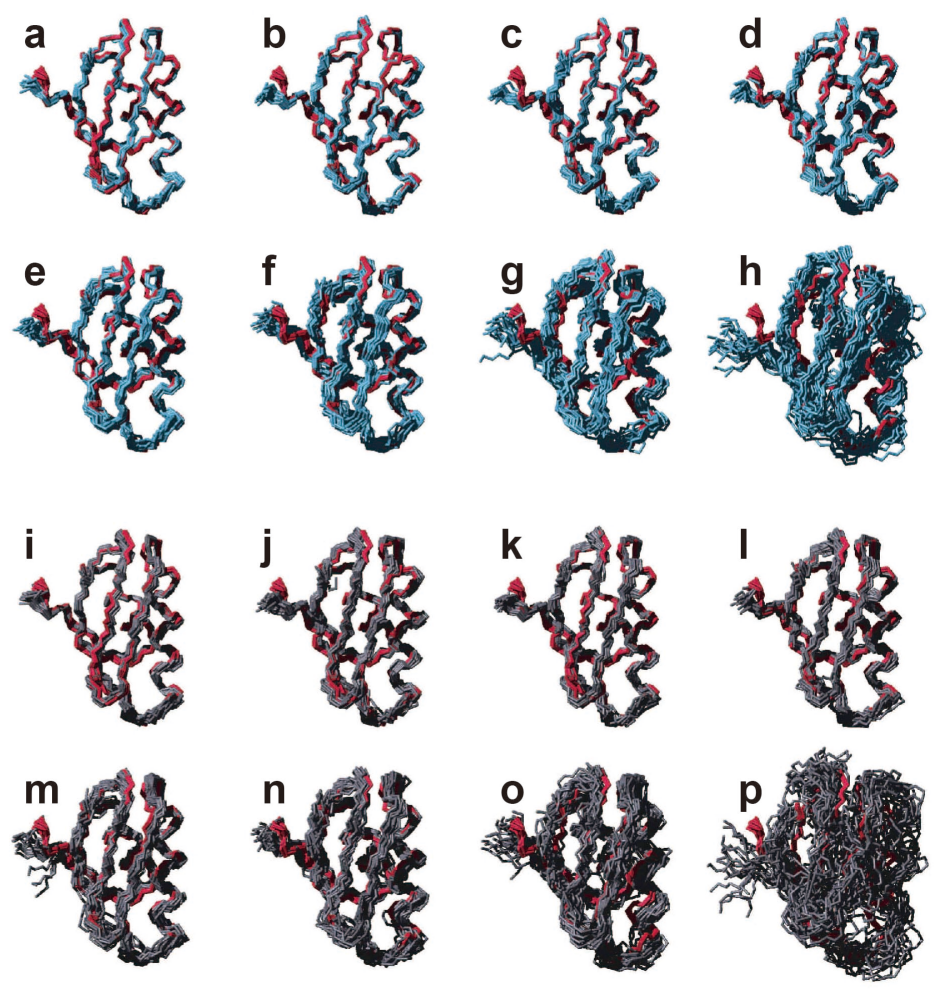